OPEN ACCESS

Edited by:

Andrew Bivard,

The University of Melbourne, Australia

Reviewed by:

Marialuisa Zedde,

Local Health Authority of Reggio

Emilia, Italy

R. Loch Macdonald,

University of Toronto, Canada

*Correspondence:

Renya Zhan

1196057@zju.edu.cn

Jianwei Pan

1202053@zju.edu.cn

${ }^{\dagger}$ These authors have contributed equally to this work

Specialty section:

This article was submitted to

Stroke,

a section of the journal

Frontiers in Neurology

Received: 02 September 2020

Accepted: 20 January 2021

Published: 18 February 2021

Citation:

Shen J, Yu J, Huang S, Mungur R, Huang K, Pan X, Yu G, Xie Z, Zhou L,

Liu Z, Cheng D, Pan J and Zhan $R$ (2021) Scoring Model to Predict Functional Outcome in Poor-Grade

Aneurysmal Subarachnoid Hemorrhage.

Front. Neurol. 12:601996. doi: 10.3389/fneur.2021.601996

\section{Scoring Model to Predict Functional Outcome in Poor-Grade Aneurysmal Subarachnoid Hemorrhage}

\author{
Jie Shen ${ }^{1 \dagger}$, Jianbo Yu ${ }^{1+}$, Sicong Huang ${ }^{1}$, Rajneesh Mungur ${ }^{1}$, Kaiyuan Huang ${ }^{1}$, Xinfa Pan ${ }^{1}$, \\ Guofeng Yu ${ }^{2}$, Zhikai Xie ${ }^{1}$, Lihui Zhou ${ }^{1}$, Zongchi Liu ${ }^{1}$, Dexin Cheng ${ }^{1}$, Jianwei Pan ${ }^{1 *}$ and \\ Renya Zhan ${ }^{1 *}$ \\ ${ }^{1}$ Department of Neurosurgery, College of Medicine, The First Affiliated Hospital, Zhejiang University, Hangzhou, China, \\ ${ }^{2}$ Department of Neurosurgery, Quzhou People's Hospital, Quzhou, China
}

Background: Patients with poor-grade aneurysmal subarachnoid hemorrhage (aSAH), defined as World Federation of Neurosurgical Societies (WFNS) grades IV-V have high rates of disability and mortality. The objective of this study was to accurately prognosticate the outcomes of patients with poor-grade aSAH by developing a new scoring model.

Methods: A total of 147 poor-grade aSAH patients in our center were enrolled. Risk variables identified by multivariate logistic regression analysis were used to devise a scoring model (total score, 0-9 points). The scores were estimated on the basis of $\beta$ coefficients. A cohort of 68 patients from another institute was used to validate the model.

Results: Multivariate logistic regression analysis revealed that modified Fisher grade $>2$ [odds ratio [OR], 2.972; $P=0.034$ ], age $\geq 65$ years (OR, 3.534; $P=0.006)$, conservative treatment (OR, 5.078; $P=0.019)$, WFNS grade $\vee(\mathrm{OR}, 2.638 ; P=0.029)$, delayed cerebral ischemia (OR, 3.170; $P=0.016$ ), shunt-dependent hydrocephalus (OR, 3.202; $P=0.032)$, and cerebral herniation (OR, 7.337; $P<0.001)$ were significant predictors for poor prognosis [modified Rankin Scale [mRS] $\geq 3$ ]. A scoring system was constructed by the integration of these factors and divided the poor-grade aSAH patients into three categories: low risk (0-1 points), intermediate risk (2-3 points), and high risk (4-9 points), with predicted risks of poor prognosis of 11,52 , and $87 \%$, respectively $(P<0.001)$. The area under the curve in the derivation cohort was 0.844 (95\% Cl, 0.778-0.909). The AUC in the validation cohort was 0.831 (95\% Cl, 0.732-0.929).

Conclusions: The new scoring model can improve prognostication and help decision-making for subsequent complementary treatment in patients with aSAH.

Keywords: scoring system, prognosis, poor-grade, aneurysmal subarachnoid hemorrhage, model

\section{INTRODUCTION}

Intracranial aneurysms are abnormal protrusions of the intracranial arterial wall arising from various causes $(1,2)$. The prevalence rate of intracranial aneurysms in the global population (mean age, 50 years) is up to $3.2 \%$ (3). A previous report described that approximately, $1-2 \%$ of these aneurysms will rupture (4). According to statistics, 
the global incidence of aneurysmal subarachnoid hemorrhage (aSAH) is $9-11$ per 100,000 people/year. Furthermore, poorgrade aSAH [World Federation of Neurological Surgeons [WFNS] grades IV-V] accounts for $18-30 \%$ of all aSAH cases (5, 6). A meta-analysis by Han et al. (7) reported a $26 \%$ mortality rate for poor-grade aSAH. At present, most related literature indicates that the disability rate for poor-grade aSAH exceeds $60 \%$ (8).

Regarding prognosis prediction, the International Subarachnoid Aneurysm Trial (ISAT) could achieve an accurate prediction of 60-day mortality after aSAH (9). Meanwhile, the Subarachnoid Hemorrhage International Trialists (SAHIT) model successfully predicted long-term outcomes and was used to counsel patients with aSAH and their family members (10). An external validation of the SAHIT model using the Barrow Ruptured Aneurysm Trial (BRAT) cohort revealed that its area under the curve (AUC) for unfavorable outcomes was 0.734 (11). It is worth pointing out that these studies included patients exposed to different subgroups of various treatment procedures, and that most of them were good-grade aSAH patients eligible for surgical treatment. Although good-grade and poor-grade aSAH patients differ in disease progression and survival prognosis $(5,12)$, previous studies typically combined these patients for analysis without detailed stratification (9-11). Therefore, the previous predictive models have some limitations for the accurate prediction of outcomes in poor-grade aSAH patients. The objective of the present study was to devise a new scoring system that can evaluate the prognosis of patients with poor-grade aSAH intuitively.

\section{MATERIALS AND METHODS}

\section{Study Design}

The derivation cohort comprised poor-grade aSAH patients who were treated in the Department of Neurosurgery at our center from January 2013 to January 2019. The validation cohort was composed of aSAH patients treated in the Department of Neurosurgery at another institute from January 2016 to January 2019. The inclusion criteria were: (1) aSAH diagnosed by computed tomography (CT) or lumbar puncture in the medical center; (2) aneurysm confirmed as the cause of SAH on digital subtraction angiography (DSA), three-dimensional CT angiography, or magnetic resonance angiography; (3) WFNS grade IV and V; (4) signed informed consent from family members of patients to cooperate with clinical treatment procedures; and (5) patients without surgical treatment in referral centers. The exclusion criteria were: (1) traumatic, mycotic,

\footnotetext{
Abbreviations: aSAH, Aneurysmal subarachnoid hemorrhage; WFNS, World Federation of Neurosurgical Societies; OR, Odds ratio; CI, Confidence interval; ICU, Intensive care unit; DCI, Delayed cerebral ischemia; CVS, Cerebral vasospasm; GOS, Glasgow Outcome Scale; IVH, Intraventricular hemorrhage; $\mathrm{ICH}$, Intracerebral hemorrhage; CT, Computed tomography; mRS, Modified Rankin score; ISAT, International Subarachnoid Aneurysm Trail; SAHIT, Subarachnoid Hemorrhage International Trialists; BRAT, Barrow Ruptured Aneurysm Trial; CLSD, Continuous lumber subarachnoid drainage; AUC, Area under the curve; WBC, White blood cell count; SDH, Shunt-dependent hydrocephalus; PASHPSS, Poor-Grade Aneurysmal Subarachnoid Hemorrhage Prognostic Scoring System.
}

or arteriovenous malformation-related aneurysms or SAH of unknown etiology; (2) WFNS grade less than or equal to III; (3) absence of important medical information for patients; and (4) patients treated with medical instruments or drugs that were not approved. The STROBE statement guideline has been implemented in this manuscript.

\section{Clinical Therapeutic Protocol}

All patients admitted under emergency conditions received early resuscitation, early CT angiography, multidisciplinary consensus consultation, conservative treatment, or surgical treatment. A multidisciplinary team of neurosurgeons and anesthesiologists made therapeutic decisions on the basis of the clinical conditions and family members' consent. The treatment mode in our study was divided into two categories: (1) the conservative group: patients who received pure medicinal conservative treatment or patients who received other basic surgical methods without treatment of the underlying aneurysm, such as external drainage surgery, hematoma evacuation, and decompressive craniectomy; and (2) the clipping or coiling group: patients who underwent primary aneurysm embolization or clipping alone, or combined with a basic surgical operation involving coiling or clipping. Patients underwent surgical treatment in accordance with an early treatment strategy (within $72 \mathrm{~h}$ ). All aSAH patients were treated with routine $\mathrm{SAH}$ treatments, including mannitol, anticonvulsants, triple-H (hypervolemia/hypertension/hemodilution) treatment, and nimodipine treatment. Antiplatelets were administered to prevent thrombosis after stent-assisted embolization.

\section{Clinical Data and Variable Definitions}

The clinical variables were collected retrospectively from the hospital database. Patient's baseline information and imaging information were collected by two doctors separately, and any conflicting items were evaluated again by a senior doctor. Age was divided into two subcategories in accordance with the cutoff age of 65 years. The modified Fisher grade was divided into two subcategories in accordance with the cutoff value of grade 2. A wide-necked aneurysm was defined as an aneurysm with a neck width $\geq 4 \mathrm{~mm}$ or a neck ratio exceeding 1:2. Cerebral herniation was diagnosed based on $\mathrm{CT}$ results and corresponding signs, including deterioration of consciousness disturbance, some focal signs, oculomotor palsy, respiratory distress, and decorticate or decerebrate rigidity (13). Among the complications, shunt-dependent hydrocephalus $(\mathrm{SDH})$ was defined as clinical deterioration occurring on the 14 th day after aSAH and no other causes were found except for hydrocephalus, at the same time, it was observed in CT that the ventricular size progressively increased and the Evans index exceeded 0.30 (14). Epilepsy was defined as rhythmic jerking, with or without preceding tonic spasms, that was focal or generalized in nature, with or without loss of consciousness. Even one late seizure was considered to be post-stroke epilepsy $(15,16)$. Aneurysm rebleeding was defined as a sudden clinical deterioration accompanied by increased subarachnoid, intracerebral, or ventricular blood flow on subsequent CT scans (17). Cerebral vasospasm (CVS) was defined as arterial stenosis 
496 aSAH patients: Diagnosed by CT or lumbar puncture; Without surgical treatment in local hospital

Excluded:

281 WFNS grade I-III ruptured intracranial aneurysms

22 Spindel or dissecting aneurysms

20 Lost to follow-up

17 Without important image records

9 Emergency death

147 patients: WFNS grade IV-V ruptured aneurysms

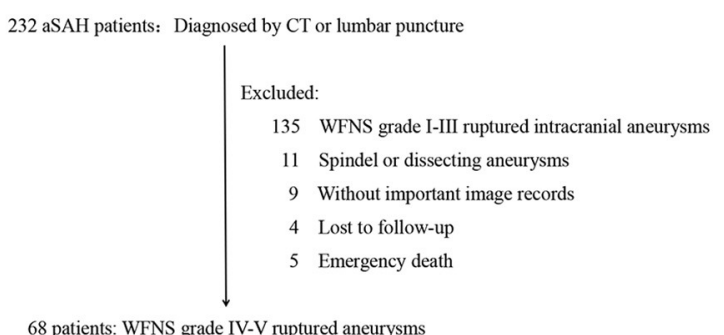

68 patients: WFNS grade IV-V ruptured aneurysms

FIGURE 1 | Study flow diagram.

found on the CT angiography examination when the patient's clinical symptoms deteriorated, or vasospasm was detected during DSA (18). Delayed cerebral ischemia (DCI) was defined as: (1) occurrence of focal neurological impairment or decrease of $\geq 2$ points on the Glasgow Coma Scale that could not be attributed to another cause, such as cerebral rebleeding or encephaledema; or (2) a new low-density area not seen on the previous CT scan and not attributable to other causes such as surgical treatment, or a low-density shadow after absorption of a hematoma (19).

\section{Outcome Measures}

A dynamic follow-up evaluation was performed at 6 months after discharge by neurosurgeons in accordance with the modified Rankin score (mRS) via telephone call or outpatient appointment. The assessment of neurological prognosis mainly focused on whether or not the patients presented with self-care ability. Functional prognosis was classified as good (mRS scores $0-2$ ) or poor (mRS scores 3-6).

\section{Statistical Analysis}

Data were analyzed using the SPSS Version 23.0 software (IBM, Armonk, NY). Continuous variables were reported as mean \pm standard deviation and compared between favorable and poor outcomes using an unpaired $t$-test. Categorical variables were reported as proportion and percentile and analyzed by the chi-square or Fisher exact test, as appropriate. Univariate and multivariate logistic regression analyses were performed using poor outcomes as the outcome variable in the derivation cohort. Variables with $P \leq 0.1$ in the univariate analyses were entered into the multivariate logistic regression analysis with stepwise backward selection. Risk variables independently associated with prognosis were entered into the new scoring model. The points for individual factors were assigned on the basis of their corresponding $\beta$ coefficients in the multivariate analysis. The discrimination of the prognostic model was assessed by the AUC in a receiver operating characteristic curve analysis. The Hosmer-Lemeshow goodness-of-fit test and a calibration plot were used to evaluate the calibration of the prediction model.

\section{RESULTS}

\section{Basic Information of Patients}

The detailed processes for the selection and exclusion of patients in the derivation group and validation group are shown in
Figure 1. In total, 147 patients were included in the derivation study and 68 patients were included in the validation cohort.

In the derivation cohort, 55 (37\%) patients were male and $92(63 \%)$ were female. Among these patients, the age range was $37-87$ years, the mean age was $61.3 \pm 11.5$ years, and $\sim 39 \%$ were aged $\geq 65$ years. The baseline characteristics of the 147 patients with poor-grade aSAH are presented in Table 1. In total, $124(84.3 \%)$ patients received surgical therapies including coiling (29.2\%) and clipping (55.1\%), and 23 (15.7\%) patients received conservative treatment. In addition, there was no significant statistical difference between the treatment approach and the WFNS grade $(P=0.110)$. The distribution of $\mathrm{mRS}$ scores among the 147 poor-grade aSAH patients with different treatments is shown in Figure 2A. As shown in Figures 2B-E, patients who received coiling or clipping had a better prognosis than patients who received conservative treatment, but there was no significant difference in prognosis between patients who received coiling or clipping. There were $114(77.6 \%)$ poor-grade aSAH patients with a modified Fisher grade $>2$ and 85 (57.8\%) patients with WFNS grade V. The distribution of mRS scores among the 147 poor-grade aSAH patients with different modified Fisher grades is shown in Figure 2F. The influences of different modified Fisher grades on the prognosis of patients are shown in Figures 2G-J. During the 6-month follow-up after discharge, 85 patients (58\%) had poor outcomes.

Of the 68 patients in the validation cohort, $25(36.7 \%)$ patients were aged $\geq 65$ years. A total of $44(64.7 \%)$ poorgrade aSAH patients had a modified Fisher grade $>2$ and 40 (58.8\%) patients presented with WFNS grade V. Forty-nine (72\%) patients underwent surgical therapies. At the 6-month follow-up after discharge, 38 (56\%) patients had poor outcomes. Specific data for the validation cohort are presented in Table $\mathbf{1 .}$

\section{Univariate Analyses of Poor Outcomes}

The associations between clinical variables and poor outcomes identified by univariate analyses are shown in Table 1. Poor prognosis was associated with age $\geq 65$ years $(P=0.027)$, intraventricular hemorrhage (IVH) $(P=0.005)$, WFNS grade $\mathrm{V}(P<0.001)$, conservative treatment $(P=0.009)$, modified Fisher grade $>2(P=0.001)$, emergence of cerebral herniation $(P<0.001)$, aneurysm rebleeding $(P=0.004)$, CVS $(P=$ $0.041)$, and DCI $(P=0.030)$. Medical histories of patients and data for aneurysms were not significantly correlated with clinical outcomes. 
TABLE 1 | Demographic and baseline characteristics of the study population and univariate analysis results of modeling cohorts.

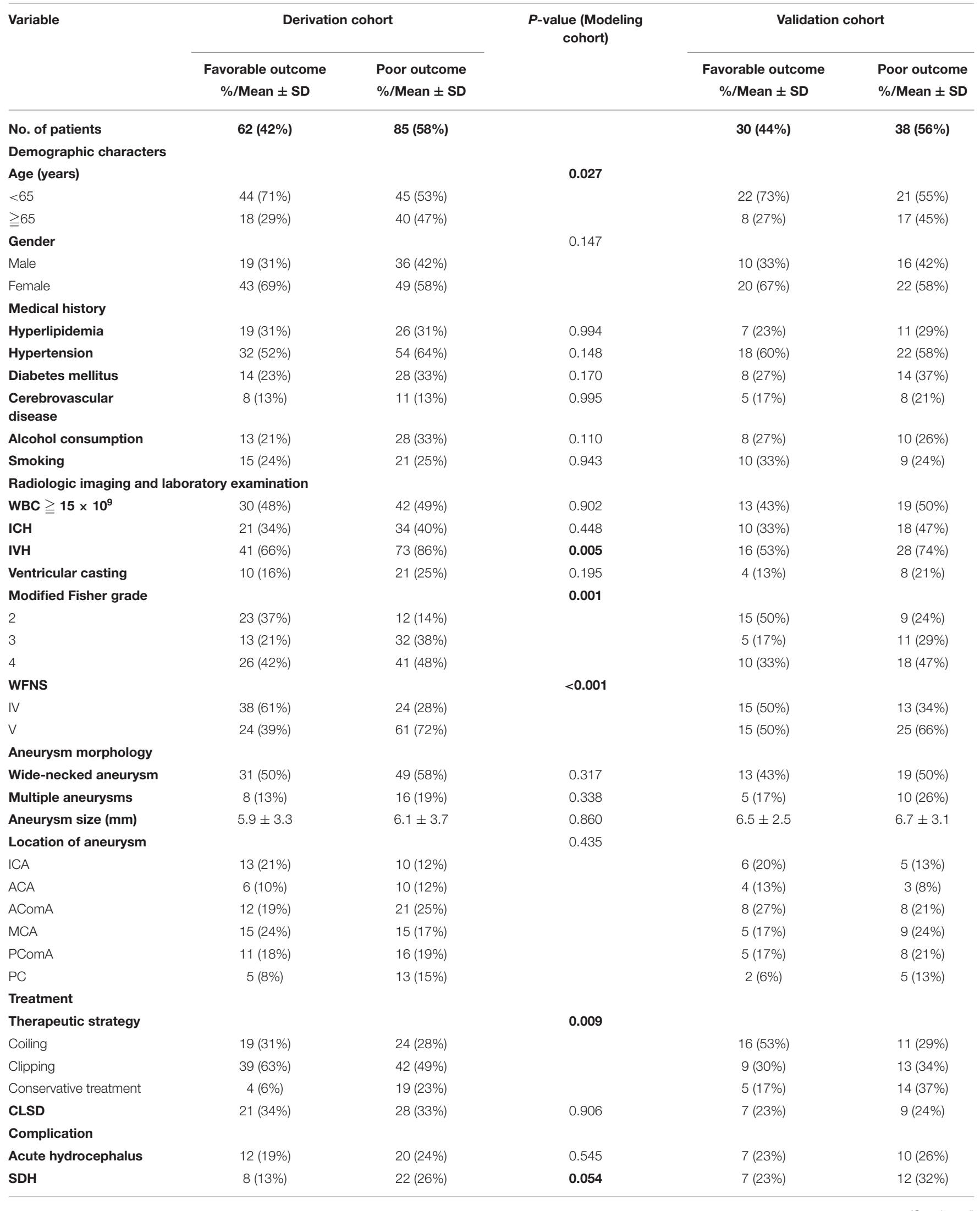


TABLE 1 | Continued

\begin{tabular}{|c|c|c|c|c|c|}
\hline \multirow[t]{2}{*}{ Variable } & \multicolumn{2}{|c|}{ Derivation cohort } & \multirow{2}{*}{$\begin{array}{l}P \text {-value (Modeling } \\
\text { cohort) }\end{array}$} & \multicolumn{2}{|c|}{ Validation cohort } \\
\hline & Favorable outcome & Poor outcome & & Favorable outcome & Poor outcome \\
\hline Aneurysm rebleeding & $1(2 \%)$ & $13(15 \%)$ & 0.004 & 0 & $8(21 \%)$ \\
\hline Epilepsy & $4(7 \%)$ & $9(11 \%)$ & 0.383 & $1(3.3 \%)$ & $3(8 \%)$ \\
\hline cvs & $7(11 \%)$ & $21(25 \%)$ & 0.041 & 7 (23\%) & $16(42 \%)$ \\
\hline $\mathrm{DCl}$ & $13(21 \%)$ & 32 (38\%) & 0.030 & $6(20 \%)$ & $11(29 \%)$ \\
\hline Cerebral herniation & $6(10 \%)$ & 38 (45\%) & $<0.001$ & $2(7 \%)$ & $16(42 \%)$ \\
\hline
\end{tabular}

ICH, intracerebral hemorrhage; IVH, intraventricular hemorrhage; WFNS, world federation of neurosurgical societies; CLSD, continuous lumbar subarachnoid drainage; SDH, shuntdependent hydrocephalus; CVS, cerebral vasospasm; DCI, delayed cerebral ischemia; ICA, internal carotid artery; ACA, anterior cerebral artery; AComA, Anterior communicating artery; MCA, middle cerebral artery; PComA, posterior communicating artery; PC, posterior cerebral circulation. $P$ values less than 0.1 are shown in bold.

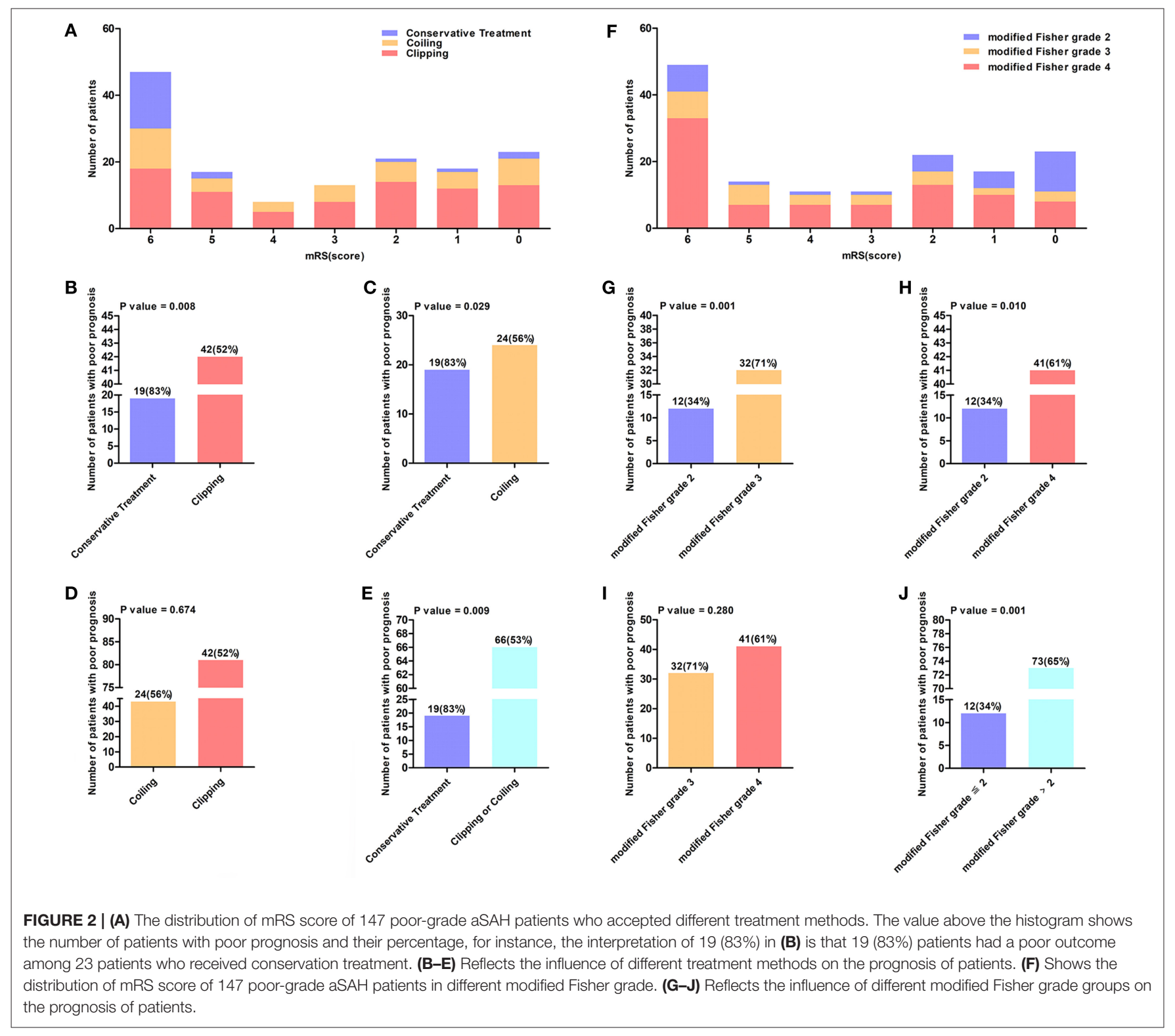


TABLE 2 | Multivariate logistic regression model for poor prognosis risk of poor-grade aSAH.

\begin{tabular}{lcccc}
\hline Variable included in model & S.E & OR & $\mathbf{9 5 \%} \mathbf{C l}$ & $\boldsymbol{P}$ \\
\hline Modified Fisher grade (grade 3, 4) & 0.515 & 2,972 & $1.083-8.156$ & 0.034 \\
Age ( $\geq$ 65) & 0.457 & 3.534 & $1.442-8.662$ & 0.006 \\
Therapeutic strategy (conservation) & 0.694 & 5.078 & $1.303-19.790$ & 0.019 \\
WFNS (grade V) & 0.444 & 2.638 & $1.104-6.300$ & 0.029 \\
DCl & 0.478 & 3.170 & $1.242-8.090$ & 0.016 \\
SDH & 0.542 & 3.202 & $1.107-9.263$ & 0.032 \\
Cerebral herniation & 0.565 & 7.337 & $2.426-22.192$ & $<0.001$ \\
Hosmer and Lemeshow test & & & & \\
$\quad X^{2}$ & & & & 6.525 \\
Degree of freedom & & & & 8.589 \\
$P$ & & & & \\
\hline
\end{tabular}

TABLE 3 | Poor-Grade Aneurysmal Subarachnoid Hemorrhage Prognostic Scoring System (PASHPSS) derived from the $\beta$ coefficients.

\begin{tabular}{|c|c|c|c|}
\hline Variable included in model & Categories & $\beta$ coefficient & Score \\
\hline \multicolumn{4}{|l|}{ Modified Fisher grade } \\
\hline & Below grade 2 & 0 (reference) & 0 \\
\hline & Grade 3, 4 & 1.09 & 1 \\
\hline \multicolumn{4}{|l|}{ Age } \\
\hline & $<65$ & 0 (reference) & 0 \\
\hline & $\geq 65$ & 1.26 & 1 \\
\hline \multicolumn{4}{|l|}{ Therapeutic strategy } \\
\hline & Coiling or clipping & 0 (reference) & 0 \\
\hline & Conservation & 1.63 & 2 \\
\hline \multicolumn{4}{|l|}{ WFNS } \\
\hline & Grade IV & 0 (reference) & 0 \\
\hline & Grade V & 0.97 & 1 \\
\hline \multicolumn{4}{|l|}{$\mathrm{DCl}$} \\
\hline & Non-DCl & 0 (reference) & 0 \\
\hline & $\mathrm{DCl}$ & 1.15 & 1 \\
\hline \multicolumn{4}{|l|}{$\mathrm{SDH}$} \\
\hline & Non-SDH & 0 (reference) & 0 \\
\hline & $\mathrm{SDH}$ & 1.16 & 1 \\
\hline \multicolumn{4}{|l|}{ Cerebral herniation } \\
\hline & Non-cerebral herniation & 0 (reference) & 0 \\
\hline & Cerebral herniation & 1.99 & 2 \\
\hline
\end{tabular}

\section{Multivariate Regression Analysis of Poor Outcome}

Ten variables with $P<0.1$ in the univariate analyses were entered into the multivariate logistic regression analysis (Table 2). The results showed that age $\geq 65$ years (OR, 3.534; $P=0.006)$, modified Fisher grade $>2(\mathrm{OR}, 2,972 ; P=0.034)$, cerebral herniation (OR, 7.337; $P<0.001)$, WFNS grade $\mathrm{V}$ (OR, 2.638; $P=0.029)$, SDH (OR, 3.202; $P=0.032)$, conservative treatment (OR, 5.078; $P=0.019)$, and DCI (OR, 3.170; $P=0.016$ ) were independent risk factors for poor outcomes. The HosmerLemeshow test reflected a satisfactory degree of consistency
TABLE 4 | Risk of poor prognosis for low, intermediate, and high-risk individuals according to the PASHPSS risk score.

\begin{tabular}{lcccc}
\hline $\begin{array}{l}\text { Risk } \\
\text { stratification }\end{array}$ & Score & $\begin{array}{c}\text { Observed risk } \\
\text { (validation } \\
\text { cohort) }\end{array}$ & Predicted risk & OR (95\% Cl) \\
\hline $\begin{array}{l}\text { Low risk } \\
\text { stratification }\end{array}$ & $0-1$ & $19 \%$ & $11 \%$ & 1 (reference) \\
$\begin{array}{l}\text { Moderate risk } \\
\text { stratification }\end{array}$ & $2-3$ & $48 \%$ & $51 \%$ & $8.6(2.2-18.7)$ \\
$\begin{array}{l}\text { High risk } \\
\text { stratification }\end{array}$ & $4-9$ & $81 \%$ & $87 \%$ & $54.2(13.2-221.9)$ \\
\hline
\end{tabular}

between the predicted risk of the model and the actual risk ( $P$ $=0.589$; Table 2).

\section{Development of the Scoring System}

By integration of the seven independent risk factors, namely modified Fisher grade $>2$, age $\geq 65$ years, conservative treatment, WFNS grade V, DCI, SDH, and cerebral herniation, a scoring system designated Poor-Grade Aneurysmal Subarachnoid Hemorrhage Prognostic Scoring System (PASHPSS) was constructed (Table 3). On the basis of the $\beta$ coefficients in the multivariate analysis, scores of 2 were assigned to cerebral herniation and conservative treatment, and scores of 1 were assigned to the other five risk factors; otherwise, a score of 0 points was assigned. In accordance with the sum of the scores (range, 0-9), the new model divided poor-grade aSAH patients into three prognostically different categories (Table 4): low risk category, $11 \%$ prediction risk of poor prognosis in patients with total scores of $0-1$ point; intermediate risk category, $51 \%$ prediction risk of poor prognosis in patients with total scores of 2-3 points; high risk category, $87 \%$ prediction risk of poor prognosis in patients with total scores of $\geq 4$ points.

\section{Discrimination and Calibration of the Scoring System}

In the derivation cohort, the AUC of the PASHPSS was 0.844 (95\% CI: 0.778-0.909; Figure 3), and the Hosmer-Lemeshow test showed good calibration $(P=0.589)$. In the validation cohort, the PASHPSS also showed good discrimination with an AUC of 0.831 (95\% CI, 0.732-0.929; Figure 3) and good calibration by the Hosmer-Lemeshow test $(P=0.984)$. Also in the validation cohort, the observed risks in the three risk categories were close to the predicted risks (Table 3): low risk category, actual observed risk of poor prognosis was 19\%; intermediate risk category, actual observed risk of poor prognosis was $48 \%$; high risk category, actual observed risk of poor prognosis was $81 \%$.

\section{DISCUSSION}

As a serious cerebrovascular disease, poor-grade aSAH has high rates of mortality and disability. In this study, the rates of poor prognosis of patients in the modeling cohort and validation cohort both exceeded 55\%. Although active and effective treatments can be provided, some aSAH patients still 

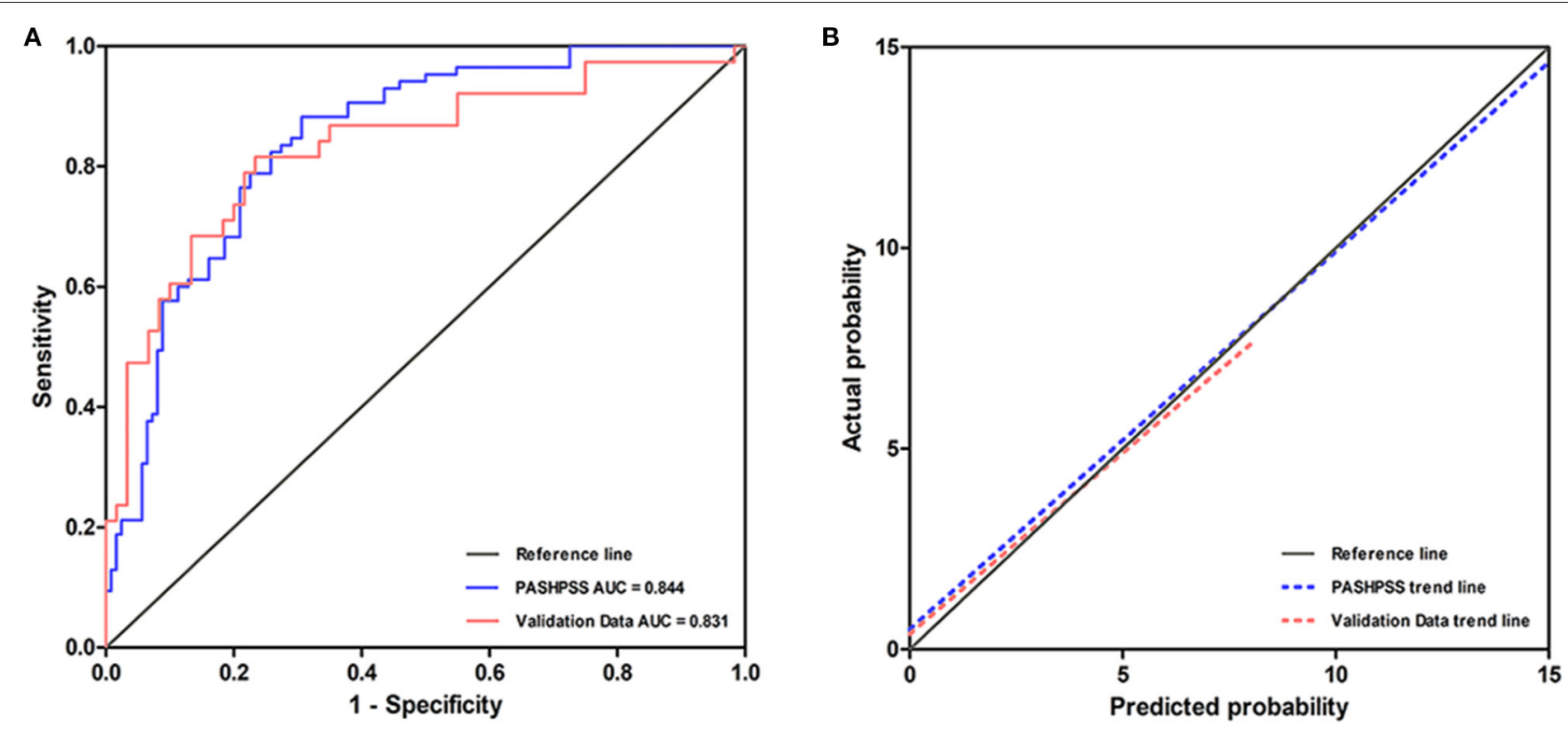

FIGURE 3 | (A) The AUC of the PASHPSS is 0.844 (95\% Cl, 0.778-0.909) in our center's derivation data, while it is 0.831 (95\% Cl, 0.732-0.929) in validation data. (B) A slope of 1 (45 degrees) with an intercept of 0 represents perfect calibration, the deviation from the reference line is smaller, the calibration is better. PASHPSS has a good calibration in derivation cohort and validation cohort.

present with neurological dysfunctions and life disorders that have great impacts on society and family members $(1,4,6,8)$. It is necessary to explore the relevant risk factors and evaluate the prognosis of these patients. Several modifiable and nonmodifiable risk factors for poor prognosis in poor-grade aSAH patients are currently known, with the most common risk factors being elderly age, cerebral herniation, WFNS grade V, and higher modified Fisher grade $(9,10,12,20-24)$. These risk factors were also identified in the present study.

The choice of treatment method is significantly related to the prognosis of patients with poor-grade aSAH. In a systematic review of 815 patients with aSAH, researchers reported that the rates of good prognosis in patients with clipping, embolization, and conservative treatment were 45.3, 36.3 , and $9.0 \%$, respectively (25). In our study, the rate of poor prognosis in patients with clipping or coiling was significantly lower than that in patients with conservative treatment, but there was no statistically significant difference in prognosis between patients with clipping or embolization. Combining our center's experience with previous literature on poor-grade aSAH patients, more aggressive treatment of the underlying aneurysm by surgery is associated with a better therapeutic prognosis than conservative treatment.

Post-operative complications play important roles in the prognosis of poor-grade aSAH patients. As a critical complication, aneurysmal rebleeding usually causes a sharp increase in intracranial pressure, damages the nerve function, and increases the risk of death in the short term (26-28). CVS is generally considered a risk factor for poor prognosis. However, immediate vasospasm is usually difficult to detect, and nimodipine is routinely used in clinical practice to prevent its occurrence, leading to an overall reduction in the incidence of CVS (29). A more commonly observed and easily detected complication during clinical treatment is DCI caused by CVS, which is a strong independent risk factor for poor prognosis in patients with poor-grade aSAH $(19,30,31)$. DCI continues to be an important cause of cognitive impairment and disability after aSAH despite aggressive management (32-34). A single-center study on 888 aSAH patients found that SDH was a strong independent risk factor for unfavorable functional outcomes (35). Our final results confirmed predictive roles for the above-mentioned factors.

Some other risk factors have also been raised in recent articles, but have not been widely recognized. IVH was considered as a risk factor for poor outcomes in many reports (36). IVH was identified in our univariate analyses, but subsequently eliminated in the multivariate regression analysis. The possible reason may be that IVH caused impairment of cerebrospinal fluid absorption by blocking arachnoid villi and brain capillaries, thereby affecting the prognosis by developing into chronic hydrocephalus $(37,38)$. Whether or not aneurysm location and size are predictive factors for poor prognosis of aSAH patients remains inconclusive (24). These inconsistent results may be explained by treatment selection biases in different studies. In the present study, there was no correlation between aneurysm location and size and long-term prognosis. In a multicenter study on poor-grade aSAH patients, Zhao et al. (21) demonstrated that wide-necked aneurysms and post-operative pneumonia were also poor prognostic factors. However, these two risk factors were not identified in our study. Leukocytosis (WBC $>15 \times 10^{9} / \mathrm{L}$ ) was regarded as a predictive factor for poor prognosis in a 9-year cohort study (22), but was not reported in other articles.

Although the current literature on poor-grade aSAH patients has focused on reporting risk factors for prognosis, prognostic predictive models for poor-grade aSAH patients are rare. A recent systematic review assessed 11 clinical prediction models 
for aSAH patients and found that the most common factors associated with outcomes were age ( 8 of 11 studies), neurologic grade on admission (10 of 11 studies), and amount of blood detected by CT examination on admission (6 of 11 studies) (24). Although the WFNS and modified Fisher grade scales were commonly used, both scales are not completely reliable in patients because of the subjective nature of the parameters on which the models were built (39). For example, the WFNS and Hunt-Hess scales are generally unreliable in intubated patients. Furthermore, in two articles that established predictive scores in poor-grade aSAH patient populations, the factors were applied, but no additional risk factors were added to circumvent the errors caused by the inter-rater and intra-rater variabilities $(22,23)$. Undeniably, more valuable risk variables added into a risk score can improve its predictivity. Treatment methods, $\mathrm{SDH}$, and DCI are three factors that affect the long-term neurological prognosis and cognitive impairment of patients, and their roles in predicting the prognosis of patients are worthy of recognition $(19,35,37,40)$. Our PASHPSS showed significantly improved discrimination compared with other risk scores by including these risk factors. For example, the AUC of the SAHIT model was 0.734 (11), while the AUC of the WAP score for poor-grade aSAH patients was 0.74 (23). Meanwhile, the AUC of the PASHPSS was 0.844 , which can be regarded as excellent, especially when predicting the prognosis of poor-grade aSAH patients.

At present, several studies have proposed prognosis models for poor-grade aSAH patients, but most of these models have limitations in reporting calibration, discrimination, and external validation. Clinicians generally do not use existing models for the prediction of prognosis in poor-grade aSAH patients, even though their internal effectiveness is not inferior to the PASHPSS (22-24), partly because they lack external validity. However, the PASHPSS showed good discrimination in the validation data. Specifically, its AUC was 0.831, meaning that the system still performed well when it was applied to a new patient cohort.

The present study shows that the PASHPSS developed with identified risk factors can predict the future risk of poor prognosis in aSAH patients very well. Furthermore, it can help guide clinical decisions and patient consultations, and may also reduce the cost of treatment by ensuring effective resource allocation. Such benefits may be particularly important in the management of patients with poor-grade aSAH.

\section{LIMITATIONS}

Some limitations of our risk score need to be discussed. First, the statistical data were retrospectively collected. Second, the results of the study only represent the subgroup of poor-grade aSAH patients. Therefore, the scoring model is applicable to the prediction of poor prognosis among poor-grade aSAH patients only. With regard to functional neurologic outcomes, we selected 6 months after discharge as the follow-up point on the basis of the critical period for neurological recovery. However, if data on long-term follow-up can be acquired, the prediction of prognosis will be more accurate. Furthermore, the modeling data were acquired from a single center, which may lead to some inevitable bias in the analysis and conclusions.

\section{CONCLUSIONS}

The obtained results have allowed us to draw the following conclusions. The main risk factors affecting the prognosis of patients with poor-grade aSAH are modified Fisher grade, elderly age, therapeutic schedule, WFNS grade, DCI, SDH, and cerebral herniation. The PASHPSS is an efficient tool for predicting the prognosis of poor-grade aSAH, can be easily measured, and is helpful for decision-making on subsequent complementary treatment and in reducing the cost of treatment by ensuring effective resource allocation.

\section{DATA AVAILABILITY STATEMENT}

The raw data supporting the conclusions of this article will be made available by the authors, without undue reservation.

\section{ETHICS STATEMENT}

Ethics approval has been obtained from the ethics committee of First Affiliated Hospital of Zhejiang University. Non-essential identifiable details have been omitted from all manuscripts. The patients next of kin provided written informed consent to participate in this study.

\section{AUTHOR CONTRIBUTIONS}

JS and JY contributed to writing the manuscript, acquisition of the data, and analysis and interpretation of the data. $\mathrm{SH}$ contributed to the acquisition of follow-up data and preliminary revision of the manuscript content. RM corrected the English language used in the manuscript. $\mathrm{KH}$ contributed to the acquisition of the data and preliminary revision of the manuscript content. XP contributed to preliminary revision of the manuscript content. GY provided the external validation data. ZX, LZ, ZL, and DC contributed to the literature review. JP and RZ contributed to the critical revision of the manuscript for intellectual content. All authors contributed to the article and approved the submitted version.

\section{FUNDING}

This research was funded by the Key Research \& Development (R\&D) Plan of Zhejiang Province (No. 2019C03034).

\section{ACKNOWLEDGMENTS}

Thanks for the data provided by $\mathrm{An} \mathrm{Wu}$, director of the Department of Neurosurgery of The Quzhou Municipal People's Hospital. This manuscript has been released as a pre-print at Research Square (41). 


\section{REFERENCES}

1. De Rooij NK, Linn FH, Van Der Plas JA, Algra A, Rinkel GJ. Incidence of subarachnoid haemorrhage: a systematic review with emphasis on region, age, gender and time trends. J Neurol Neurosurg Psychiatry. (2007) 78:1365-72. doi: 10.1136/jnnp.2007.117655

2. Wiebers D, Whisnant JP, Huston J, Meissner I, Brown RD, Piepgras DG, et al. Unruptured intracranial aneurysms: natural history, clinical outcome, and risks of surgical and endovascular treatment. Lancet. (2003) 362:103-10. doi: 10.1016/S0140-6736(03)13860-3

3. Thompson BG, Brown RD Jr, Amin-Hanjani S, Broderick JP, Cockroft $\mathrm{KM}$, Connolly ES, et al. Guidelines for the management of patients with unruptured intracranial aneurysms: a guideline for healthcare professionals from the American Heart Association/American Stroke Association. Stroke. (2015) 46:2368-400. doi: 10.1161/STR.0000000000000070

4. Ellis JA, Nossek E, Kronenburg A, Langer DJ, Ortiz RA. Intracranial aneurysm: diagnostic monitoring, current interventional practices, and advances. Curr Treat Options Cardiovasc Med. (2018) 20:94. doi: 10.1007/s11936-018-0695-y

5. Steiner T. Juvela S, Unterberg A, Jung C, Forsting M, Rinkel G, et al. European stroke organization guidelines for the management of intracranial aneurysms and subarachnoid haemorrhage. Cerebrovasc Dis. (2013) 35:93112. doi: $10.1159 / 000346087$

6. Howard. BM, Barrow. DL. Outcomes for patients with poor-grade subarachnoid hemorrhage: to treat or not to treat? World Neurosurgery. (2016) 85:125-9. doi: 10.1016/j.wneu.2015.10.034

7. Han Y, Ye F, Long X, Li A, Xu H, Zou L, et al. Ultra-Early treatment for poor-grade aneurysmal subarachnoid hemorrhage: a systematic review and meta-analysis. World Neurosurg. (2018) 115:e160-71. doi: 10.1016/j.wneu.2018.03.219

8. Wartenberg KE. Critical care of poor-grade subarachnoid hemorrhage. Curr Opin Crit Care. (2011) 17:85-93. doi: 10.1097/MCC.0b013e3283 $42 \mathrm{f} 83 \mathrm{~d}$

9. Risselada R, Lingsma HF, Bauer-Mehren A, Friedrich CM, Molyneux AJ, Kerr RS, et al. Prediction of 60 day case-fatality after aneurysmal subarachnoid haemorrhage: results from the International Subarachnoid Aneurysm Trial (ISAT). Eur J Epidemiol. (2010) 25:261-6. doi: 10.1007/s10654-010-9432-x

10. Jaja BNR, Saposnik G, Lingsma HF, Macdonald E, Thorpe KE, Mamdani $\mathrm{M}$, et al. Development and validation of outcome prediction models for aneurysmal subarachnoid haemorrhage: the SAHIT multinational cohort study. BMJ. (2018) 360:j5745. doi: 10.1136/bmj.j5745

11. Mascitelli JR, Cole T, Yoon S, Nakaji P, Albuquerque FC, Mcdougall CG, et al. External validation of the subarachnoid hemorrhage international trialists (SAHIT) predictive model using the barrow ruptured aneurysm trial (BRAT) cohort. Neurosurgery. (2020) 86:101-6. doi: 10.1093/neuros/nyy600

12. Molyneux A, Kerr R, Stratton I, Sandercock P, Clarke M, Shrimpton J, et al. International subarachnoid aneurysm trial (ISAT) of neurosurgical clipping versus endovascular coiling in 2143 patients with ruptured intracranial aneurysms: a randomised trial. Lancet. (2002) 360:1267-74. doi: 10.1016/S0140-6736(02)11314-6

13. Riveros Gilardi B, Munoz Lopez JI, Hernandez Villegas AC, Garay Mora JA, Rico Rodriguez OC, Chavez Appendini R, et al. Types of cerebral herniation and their imaging features. Radiographics. (2019) 39:1598-610. doi: $10.1148 / \mathrm{rg} .2019190018$

14. Suzuki H, Kinoshita N, Imanaka-Yoshida K, Yoshida T, Taki W. Cerebrospinal fluid tenascin-C increases preceding the development of chronic shunt-dependent hydrocephalus after subarachnoid hemorrhage. Stroke. (2008) 39:1610-2. doi: 10.1161/STROKEAHA.107.505735

15. Lin CL, Dumont AS, Lieu AS, Yen CP, Hwang SL, Kwan AL, et al. Characterization of perioperative seizures and epilepsy following aneurysmal subarachnoid hemorrhage. J Neurosurg. (2003) 99:978-85. doi: 10.3171/jns.2003.99.6.0978

16. Engel J Jr. Report of the ILAE classification core group. Epilepsia. (2006) 47:1558-68. doi: 10.1111/j.1528-1167.2006.00215.x

17. Schuss P, Hadjiathanasiou A, Borger V, Wispel C, Vatter H, Guresir E. Poor-Grade aneurysmal subarachnoid hemorrhage: factors influencing functional outcome-a single-center series. World Neurosurg. (2016) 85:125-9. doi: 10.1016/j.wneu.2015.08.046
18. Frontera JA, Fernandez A, Schmidt JM, Claassen J, Wartenberg KE, Badjatia N, et al. Defining vasospasm after subarachnoid hemorrhage: what is the most clinically relevant definition? Stroke. (2009) 40:1963-8. doi: 10.1161/STROKEAHA.108.544700

19. Vergouwen MD, Vermeulen M, Van Gijn J, Rinkel GJ, Wijdicks EF, Muizelaar JP, et al. Definition of delayed cerebral ischemia after aneurysmal subarachnoid hemorrhage as an outcome event in clinical trials and observational studies: proposal of a multidisciplinary research group. Stroke. (2010) 41:2391-5. doi: 10.1161/STROKEAHA.110.589275

20. Molyneux AJ, Birks J, Clarke A, Sneade M, Kerr RS. The durability of endovascular coiling versus neurosurgical clipping of ruptured cerebral aneurysms: 18 year follow-up of the UK cohort of the International Subarachnoid Aneurysm Trial (ISAT). Lancet. (2015) 385:691-7. doi: 10.1016/S0140-6736(14)60975-2

21. Zhao B, Yang H, Zheng K, Li Z, Xiong Y, Tan X, et al. Preoperative and postoperative predictors of long-term outcome after endovascular treatment of poor-grade aneurysmal subarachnoid hemorrhage. J Neurosurg. (2017) 126:1764-71. doi: 10.3171/2016.4.JNS152587

22. Szklener S, Melges A, Korchut A, Zaluska W, Trojanowski T, Rejdak R, et al. Predictive model for patients with poor-grade subarachnoid haemorrhage in 30-day observation: a 9-year cohort study. BMJ Open. (2015) 5:e007795. doi: 10.1136/bmjopen-2015-007795

23. Zheng K, Zhong M, Zhao B, Chen SY, Tan XX, Li ZQ, et al. Poor-Grade aneurysmal subarachnoid hemorrhage: risk factors affecting clinical outcomes in intracranial aneurysm patients in a multi-center study. Front Neurol. (2019) 10:123. doi: 10.3389/fneur.2019.00123

24. Jaja BN, Cusimano MD, Etminan N, Hanggi D, Hasan D, Ilodigwe $\mathrm{D}$, et al. Clinical prediction models for aneurysmal subarachnoid hemorrhage: a systematic review. Neurocrit Care. (2013) 18:143-53. doi: $10.1007 / \mathrm{s} 12028-012-9792-\mathrm{z}$

25. Ohkuma H, Shimamura N, Naraoka M, Katagai T. Aneurysmal subarachnoid hemorrhage in the elderly over age 75: a systematic review. Neurol Med Chir. (2017) 57:575-83. doi: 10.2176/nmc.ra.2017-0057

26. Van Donkelaar CE, Bakker NA, Veeger NJ, Uyttenboogaart M, Metzemaekers JD, Luijckx GJ, et al. Predictive factors for rebleeding after aneurysmal subarachnoid hemorrhage: rebleeding aneurysmal subarachnoid hemorrhage study. Stroke. (2015) 46:2100-6. doi: 10.1161/STROKEAHA.115.010037

27. Larsen CC, Astrup J. Rebleeding after aneurysmal subarachnoid hemorrhage: a literature review. World Neurosurg. (2013) 79:307-12. doi: 10.1016/j.wneu.2012.06.023

28. Lu VM, Graffeo CS, Perry A, Carlstrom LP, Rangel-Castilla L, Lanzino G, et al. Rebleeding drives poor outcome in aneurysmal subarachnoid hemorrhage independent of delayed cerebral ischemia: a propensity-score matched cohort study. J Neurosurg. (2019) 133:360-8. doi: 10.3171/2019.4.JNS19779

29. Findlay JM, Nisar J, Darsaut T. Cerebral vasospasm: a review. Can J Neurol Sci. (2016) 43:15-32. doi: 10.1017/cjn.2015.288

30. Al-Mufti F, Roh D, Lahiri S, Meyers E, Witsch J, Frey HP, et al. Ultraearly angiographic vasospasm associated with delayed cerebral ischemia and infarction following aneurysmal subarachnoid hemorrhage. J Neurosurg. (2017) 126:1545-51. doi: 10.3171/2016.2.JNS151939

31. Leclerc JL, Blackburn S, Neal D, Mendez NV, Wharton JA, Waters MF, et al. Haptoglobin phenotype predicts the development of focal and global cerebral vasospasm and may influence outcomes after aneurysmal subarachnoid hemorrhage. Proc Natl Acad Sci USA. (2015) 112:1155-60. doi: 10.1073/pnas.1412833112

32. Wang X, Han C, Xing D, Wang C, Ding X. Early management of poor-grade aneurysmal subarachnoid hemorrhage: a prognostic analysis of 104 patients. Clin Neurol Neurosurg. (2019) 179:4-8. doi: 10.1016/j.clineuro.2019.02.003

33. Etminan N, Vergouwen Md, Rl. M. Angiographic vasospasm versus cerebral infarction as outcome measures after aneurysmal subarachnoid hemorrhage. Acta Neurochirurgica. (2013) 115:33-40. doi: 10.1007/978-3-7091-1192-5_8

34. Kreiter KT, Copeland D, Bernardini GL, Bates JE, Peery S, Claassen J, et al. Predictors of cognitive dysfunction after subarachnoid hemorrhage. Stroke. (2002) 33:200-8. doi: 10.1161/hs0102.101080

35. Paisan GM, Ding D, Starke RM, Crowley RW, Liu KC. Shunt-Dependent hydrocephalus after aneurysmal subarachnoid hemorrhage: predictors and long-term functional outcomes. Neurosurgery. (2018) 83:393-402. doi: 10.1093/neuros/nyx393 
36. Nguyen HS, Li L, Patel M, Kurpad S, Mueller W. Radiodensity of intraventricular hemorrhage associated with aneurysmal subarachnoid hemorrhage may be a negative predictor of outcome. J Neurosurg. (2018) 128:1032-6. doi: 10.3171/2016.11.JNS152839

37. De Oliveira JG, Beck J, Setzer M, Gerlach R, Vatter H, Seifert V, et al. Risk of shunt-dependent hydrocephalus after occlusion of ruptured intracranial aneurysms by surgical clipping or endovascular coiling: a single-institution series and meta-analysis. Neurosurgery. (2007) 61:924-33; discussion 933-24. doi: 10.1227/01.neu.0000303188.72425.24

38. Lai L, Morgan MK. Predictors of in-hospital shunt-dependent hydrocephalus following rupture of cerebral aneurysms. J Clin Neurosci. (2013) 20:1134-8. doi: 10.1016/j.jocn.2012.09.033

39. Degen LA, Dorhout Mees SM, Algra A, Rinkel GJ. Interobserver variability of grading scales for aneurysmal subarachnoid hemorrhage. Stroke. (2011) 42:1546-9. doi: 10.1161/STROKEAHA.110.601211

40. Van Donkelaar CE, Dijkland SA, Van Den Bergh WM, Bakker J, Dippel DW, Nijsten MW, et al. Early circulating lactate and glucose levels after aneurysmal subarachnoid hemorrhage correlate with poor outcome and delayed cerebral ischemia: a two-center cohort study. Crit Care Med. (2016) 44:966-72. doi: 10.1097/CCM.0000000000001569

41. Jie S, Jianbo Y, Sicong H, Rajneesh M, Kaiyuan H, Xinfa P, et al. A Scoring Model to Predict the Prognosis of Patients With Poor-Grade Aneurysmal Subarachnoid Hemorrhage Research Square 2020. (2020) Available online at: https://researchsquare.com/article/rs-69333/v1 (accessed Sep 2, 2020).

Conflict of Interest: The authors declare that the research was conducted in the absence of any commercial or financial relationships that could be construed as a potential conflict of interest.

Copyright (c) 2021 Shen, Yu, Huang, Mungur, Huang, Pan, Yu, Xie, Zhou, Liu, Cheng, Pan and Zhan. This is an open-access article distributed under the terms of the Creative Commons Attribution License (CC BY). The use, distribution or reproduction in other forums is permitted, provided the original author(s) and the copyright owner(s) are credited and that the original publication in this journal is cited, in accordance with accepted academic practice. No use, distribution or reproduction is permitted which does not comply with these terms. 